\title{
Somatic variation as an incidental finding in the pediatric next-generation sequencing era
}

\author{
Marilena Melas, ${ }^{1}$ Mariam T. Mathew, ${ }^{1,2,3}$ Mari Mori, ${ }^{3,4}$ Vijayakumar Jayaraman, ${ }^{1}$ \\ Sarah A. Wilson, ${ }^{1}$ Cortlandt Martin, ${ }^{4}$ Amanda E. Jacobson-Kelly, ${ }^{3,5}$ Ben J. Kelly, ${ }^{1}$ \\ Vincent Magrini, ${ }^{1,3}$ Elaine R. Mardis, ${ }^{1,3}$ Catherine E. Cottrell, ${ }^{1,2,3}$ and Kristy Lee ${ }^{1,2,3}$ \\ ${ }^{1}$ The Steve and Cindy Rasmussen Institute for Genomic Medicine, Nationwide Children's Hospital, Columbus, \\ Ohio 43215, USA; ${ }^{2}$ Department of Pathology, ${ }^{3}$ Department of Pediatrics, The Ohio State University, \\ Columbus, Ohio 43210, USA; ${ }^{4}$ Genetic and Genomic Medicine, ${ }^{5}$ Division of Hematology/Oncology/BMT, \\ Nationwide Children's Hospital, Columbus, Ohio 43215, USA
}

Corresponding author: kristy.lee@nationwidechildrens org

(C) 2021 Melas et al. This article is distributed under the terms of the Creative Commons

Attribution-NonCommercial License, which permits reuse and redistribution, except for commercial purposes, provided that the original author and source are credited.

\section{Ontology terms:}

craniosynostosis; Dandy-Walker malformation; severe global

developmental delay

Published by Cold Spring Harbor Laboratory Press

doi:10.1101/mcs.a006135

\begin{abstract}
The methodologic approach used in next-generation sequencing (NGS) affords a high depth of coverage in genomic analysis. Inherent in the nature of genomic testing, there exists potential for identifying genomic findings that are incidental or secondary to the indication for clinical testing, with the frequency dependent on the breadth of analysis and the tissue sample under study. The interpretation and management of clinically meaningful incidental genomic findings is a pressing issue particularly in the pediatric population. Our study describes a 16-mo-old male who presented with profound global delays, brain abnormality, progressive microcephaly, and growth deficiency, as well as metopic craniosynostosis. Clinical exome sequencing (ES) trio analysis revealed the presence of two variants in the proband. The first was a de novo variant in the PPP2R1A gene (c.773G >A, p.Arg258His), which is associated with autosomal dominant (AD) intellectual disability, accounting for the proband's clinical phenotype. The second was a recurrent hotspot variant in the CBL gene (c.1111T > C, p.Tyr371 His), which was present at a variant allele fraction of $11 \%$, consistent with somatic variation in the peripheral blood sample. Germline pathogenic variants in CBL are associated with AD Noonan syndrome-like disorder with or without juvenile myelomonocytic leukemia. Molecular analyses using a different tissue source, buccal epithelial cells, suggest that the CBL alteration may represent a clonal population of cells restricted to leukocytes. This report highlights the laboratory methodologic and interpretative processes and clinical considerations in the setting of acquired variation detected during clinical ES in a pediatric patient.
\end{abstract}

\section{INTRODUCTION}

Exome sequencing (ES) uses next-generation sequencing (NGS) as an unbiased approach to simultaneously evaluate the protein-coding regions of all genes in the human genome. In the pediatric setting, ES has shown clinical utility in patients with suspected genetic disorders (Meng et al. 2017; Clark et al. 2018). In addition to identifying disease-associated findings primary to the indication for testing, this broad approach increases the potential for revealing incidental genomic findings that are secondary to the indication for testing. The American College of Medical Genetics and Genomics (ACMG) provides recommendations for reporting secondary findings in 73 genes deemed medically actionable (Miller et al. 2021), based on their association with cancer predisposition, cardiovascular disease, and inborn errors of 
metabolism. These secondary findings are typically germline and consequently may impact other at-risk family members.

The use of NGS has the potential to produce depths of sequencing read coverage suitable to identify somatic variation, as indicated by variants present at reduced allele frequency/fraction (VAF) from that expected in a heterozygous or hemizygous germline state. Mosaicism is defined as the presence of two or more cell populations, derived from a single fertilized egg (zygote), that are genotypically distinct (Gajecka 2016). Mosaicism is an important contributor to disease-causing variation that can be identified by ES in the pediatric setting (Cao et al. 2019; Miller et al. 2020a). Somatic mosaicism results from genomic alterations that occur postzygotically (Biesecker and Spinner 2013; Lupski 2013; Acuna-Hidalgo et al. 2015). Somatic variants may be present at relatively low VAFs, which can be challenging to detect depending upon the sequencing coverage achieved. Therefore, sequencing to higher depths can enhance the potential to identify somatic mosaicism.

Recently, ACMG published guidance for the detection of incidental acquired variants in germline genetic and genomic testing, specifically surrounding genes commonly altered in clonal hematopoiesis (Chao et al. 2021). This ACMG statement provides points for consideration surrounding test performance and data analysis, variant interpretation and reporting, and clinical interpretation and diagnosis. Genomic DNA derived from the leukocytes within peripheral blood is commonly utilized for germline genetic testing. In the setting of clinical testing, alternate sources for DNA can often include saliva or buccal cells. Notably, prior studies have demonstrated varying cellular composition among buccal and saliva samples, with variability in the percentages of leukocytes and epithelial cells related to sampling, age of individual, oral health, and other factors (Theda et al. 2018).

Although ES is useful for identifying Mendelian disorders, it is important to consider that identified variation may be unique to the sampled tissue type. Discerning the etiology of somatic variation has important implications for patient management, surveillance, diagnosis, and counseling. In this report, we discuss the interpretative, methodologic, and clinical considerations surrounding the detection of a pathogenic somatic $C B L$ variant, as well as a heterozygous likely pathogenic PPP2R1A variant, identified by clinical ES in peripheral blood from a boy with profound global delays and growth deficiency without a clinical diagnosis of Noonan syndrome or juvenile myelomonocytic leukemia (JMML).

\section{RESULTS}

\section{Clinical Presentation and Family History}

The proband is a 16-mo-old full-term male, referred to medical genetics at 8 mo of age due to prenatally noted ventriculomegaly, progressive microcephaly, and global developmental delay. Head circumference at birth was $33 \mathrm{~cm}(Z=-1.98)$, whereas later measurements showed $41 \mathrm{~cm}(8 \mathrm{mo} ; Z=-3.22)$ and $41.8 \mathrm{~cm}(3 \mathrm{yr} ; Z=-6.24)$. Postnatal brain magnetic resonance imaging (MRI) showed supratentorial volume loss and lateral ventriculomegaly, mildly enlarged third ventricle, and retrocerebellar cerebrospinal fluid (CSF) space. Head computed tomography (CT) was ordered because of an abnormal head shape and revealed metopic craniosynostosis. Other anthropometric measurement showed severe progressive growth deficiency with weights of $2.93 \mathrm{~kg}$ at birth $(Z=-1.05), 7.85 \mathrm{~kg}$ at $8 \mathrm{mo}(Z=-1.26)$, and $9.2 \mathrm{~kg}$ at $2 \mathrm{yr} 10 \mathrm{mo}(Z=-4.33)$, and lengths of $54 \mathrm{~cm}$ at birth $(Z=0.51), 69 \mathrm{~cm}$ at $8 \mathrm{mo}(Z=-0.69)$ and $84.5 \mathrm{~cm}$ at $2 \mathrm{yr} 8 \mathrm{mo}(Z=-2.46)$. The child was noted with normally shaped but low-set ears, hypotonia, joint hypermobility, and severe scoliosis. An ophthalmological evaluation showed axial hypermetropia, exotropia, and delayed visual maturation. Spinal MRI showed moderate to severe dextrorotary T9-T10 scoliosis, which was progressive on serial imaging and required casting. At 3 years of age, he continues to have profound 
Table 1. Exome sequencing (ES) trio analysis variant characteristics and classification

\begin{tabular}{|c|c|c|c|c|c|c|c|}
\hline $\begin{array}{l}\text { Gene (transcript } \\
\text { ID) }\end{array}$ & $\begin{array}{l}\text { Genomic change } \\
\text { (GRCh37) }\end{array}$ & $\begin{array}{l}\text { HGVS coding } \\
\text { reference }\end{array}$ & $\begin{array}{l}\text { HGVS protein } \\
\text { reference }\end{array}$ & $\begin{array}{l}\text { Variant } \\
\text { type }\end{array}$ & $\begin{array}{l}\text { Predicted } \\
\text { effect }\end{array}$ & $\mathrm{dbSNP}$ & $\begin{array}{l}\text { Genotype/ } \\
\text { inheritance }\end{array}$ \\
\hline $\begin{array}{l}\text { PPP2R1A } \\
\quad(\text { NM_014225.5) }\end{array}$ & $\begin{array}{l}\text { Chr 19:52716329 } \\
\quad \text { G > A }\end{array}$ & c. $773 \mathrm{G}>\mathrm{A}$ & p.R258H & Missense & $\begin{array}{l}\text { Likely } \\
\text { pathogenic }\end{array}$ & rs863225094 & $\begin{array}{l}\text { Heterozygous/de } \\
\text { novo }\end{array}$ \\
\hline $\begin{array}{l}\text { CBL } \\
\quad \text { (NM_005188.3) }\end{array}$ & $\begin{array}{l}\text { Chr 11:119148891 } \\
\quad \text { T>C }\end{array}$ & c. $1111 \mathrm{~T}>\mathrm{C}$ & p.Y371H & Missense & Pathogenic & rs267606706 & Somatic \\
\hline
\end{tabular}

global delays and swallowing issues. He is not able to crawl or sit unsupported and has no specific words, although he is able to follow some simple directions. A complete blood count with differentials has been completely normal without leukocytosis, anemia, cytopenia, or other signs of JMML. Neither parent has a history of developmental delay, learning issues, or short stature.

\section{Genomic Analyses and Variant Interpretation}

Clinical ES trio analysis was performed on genomic DNA isolated from peripheral blood of the proband and parents. This analysis identified a de novo missense variant in the PPP2R1A gene (c.773G $>A$ ), resulting in the replacement of an arginine with a histidine at amino acid 258 (p.Arg258His) (Table 1; Fig. 1) in the seventh HEAT (huntingtin-elongation-A subunitTOR) repeat motif. This variant was classified as likely pathogenic in accordance with the standards and guidelines for variant interpretation set forth by ACMG and the Association for Molecular Pathology (AMP) (Richards et al. 2015). In brief, the PPP2R1A variant was verified as a de novo change, with both maternity and paternity confirmed via in silico analysis of trio ES data and end-point polymerase chain reaction (PCR) of loci with high population minor allele frequency used to compare inherited genotypes (Miller et al. 2020a). This variant was absent from large-scale population databases (Karczewski et al. 2020), has been

A

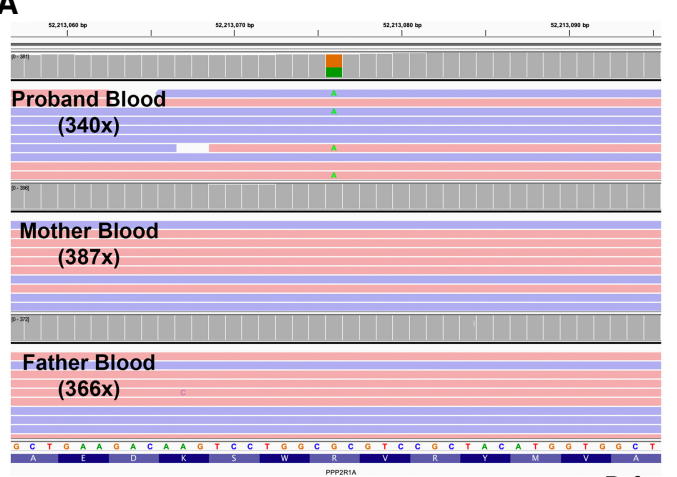

Reference: G A A G A C A A G T C C T G G C G C G T C C G C T A C A T G

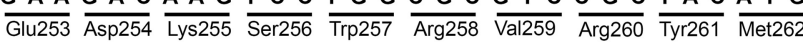

Alternate: G A A G A C A A G T C C T G G C A C G T C C G C T A C A T G $\overline{\text { Glu253 }} \overline{\text { Asp254 }} \overline{\text { Lys255 }} \overline{\text { Ser256 }} \overline{\text { Trp257 }} \overline{\text { Arg258 }} \overline{\text { Val259 }} \overline{\text { Arg260 }} \overline{\text { Tyr261 }} \overline{\text { Met262 }}$

Figure 1. PPP2R1A variant identified by ES analysis and confirmation via Sanger sequencing. (A) De novo heterozygous PPP2R1A variant visualized by the Integrative Genomics Viewer (IGV) (reference base G in orange, variant base $A$ in green) in the proband (top panel), with reference base detected at this position for samples from the mother (middle panel) and father (bottom panel). (B) Sanger sequencing analysis of the PPP2R1A variant visualized by Sequencher in the proband (top panel), mother (middle panel), and father (bottom panel) confirms the PPP2R1A variant is de novo in the proband. 
reported in the literature with supporting in vitro functional studies suggesting a reduction in protein activity (Houge et al. 2015; Ji et al. 2019) hindering binding to the regulatory subunit B55a encoded by PPP2R2A (Lenaerts et al. 2021), and is predicted to be damaging by multiple computational (in silico) functional prediction algorithms (ACMG/AMP: PS2, PM2, PM_PS3, PP3). The PPP2R1A variant was verified via orthogonal Sanger sequencing of proband and parental samples and observed only in the proband sample. This finding was considered to be diagnostic for the proband's clinical presentation.

The proband also harbored a somatic missense variant in the CBL gene (c.1111T > C) observed at a reduced VAF (11\%) in ES resulting in the substitution of a tyrosine with a histidine at amino acid position 371 (p.Tyr371 His) (Table 1; Fig. 2). Germline variants in the CBL gene are associated with autosomal dominant (AD) Noonan syndrome-like disorder with or without JMML (OMIM: 613563), a developmental disorder resembling Noonan syndrome with extensive phenotypic heterogeneity and variable expressivity (Martinelli et al. 2010). This variant was classified as pathogenic in accordance with ACMG/AMP guidelines (Richards et al. 2015). Through orthogonal studies, the CBL variant was visualized by Sanger sequencing at low level in the proband and was absent from both parents (ACMG/AMP: PS2), consistent with a somatic finding (Fig. 2). Multiple in vitro studies have associated this variant with up-regulation of critical genes in the RAS pathway and diminished E3 ligase activity

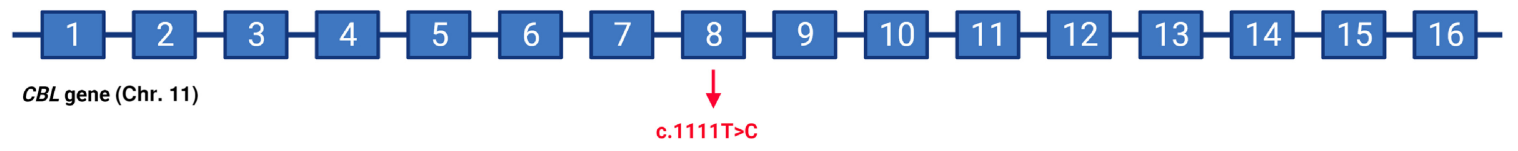

Exome and Sanger Sequencing

A

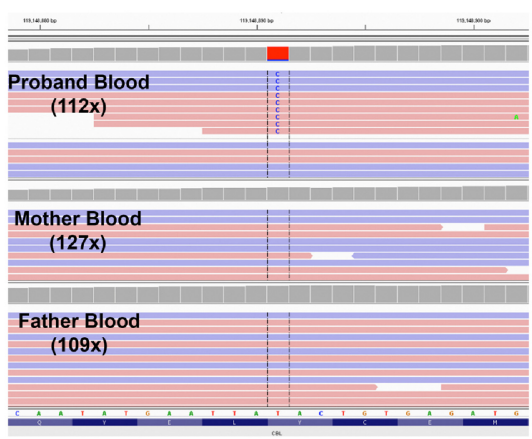

B Proband Buccal

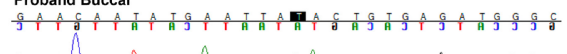
Whowhwnmmonh Proband Blood Specimen 1

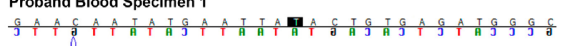
Whwownownowh Proband Blood Specimen 2 G MNWWWMWWWMWMWMa

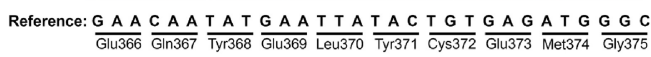
Alternate: G A A C A A T A T G A A T T A C A C T G T G A G A T G G G C $\frac{\text { Glu366 }}{\text { Gln } 367} \frac{\text { Tyr368 }}{\text { Glu369 }} \frac{\text { Leu370 }}{\text { His } 371} \frac{\text { Cys } 372}{\text { Glu3373 }} \frac{\text { A T } 374}{\text { Gly375 }}$

Targeted Next-Generation Sequencing of $C B L$ c.1111T $>C$

C

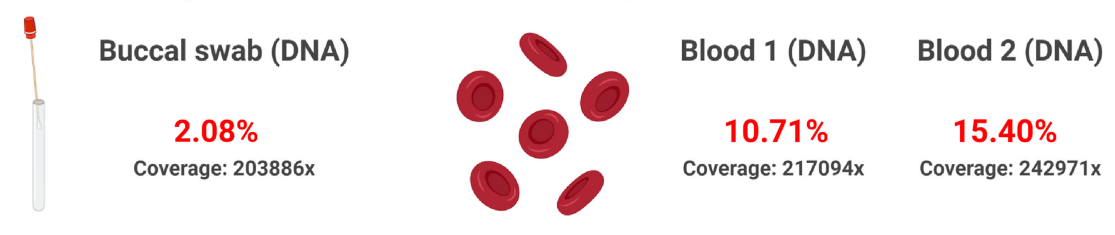

Figure 2. Identification of $C B L$ variant. Diagram of the $C B L$ gene, indicating the c.1111T $>C$ variant in exon 8. (A) Somatic heterozygous CBL variant visualized by the Integrative Genomics Viewer (IGV) (reference base $T$ in red, variant base $C$ in blue) in the proband (top panel), with reference base detected at this position for samples from the mother (middle panel) and father (bottom panel). (B) Sanger sequencing analysis of the CBL variant visualized in Sequencher in the proband's buccal epithelium, exome sequencing (ES) peripheral blood sample (blood specimen 1), and post-ES peripheral blood sample (blood specimen 2). (C) Targeted next-generation sequencing (NGS) analysis for the CBL variant in buccal cells, ES peripheral blood sample (blood 1), and post-ES peripheral blood sample (blood 2). The percentage of the CBL variant and total coverage is indicated for each sample. 
(ACMG/AMP: PS3) (Niemeyer et al. 2010; Javadi et al. 2013). This variant has been reported in multiple affected individuals with JMML and is a mutational hotspot, occurring at a conserved tyrosine kinase residue within the critical hotspot linker region of the protein (ACMG/AMP: PM1) (Kales et al. 2010; Loh et al. 2009). Last, this variant is predicted to be damaging by multiple computational (in silico) functional algorithms (ACMG/AMP: PP3).

To further discern the clinical significance of the CBL variant, subsequent sequencing analyses were performed on an alternate tissue source derived from the patient's buccal epithelium. Initial study by Sanger sequencing analysis on DNA obtained from the proband's buccal cells was negative for the $C B L$ variant, suggesting this alteration may represent a clonal population confined to the peripheral blood (Fig. 2). This result was considered incidental or secondary to the primary indications for testing. The patient was referred to hematology/ oncology for evaluation. Follow-up Sanger sequencing analysis was performed on peripheral blood obtained 12 mo post-ES analysis showed persistent presence of the CBL variant (Fig. 2).

Given the limitation in the detection of low VAF alterations by Sanger sequencing, targeted NGS analysis was performed on all available proband DNA samples including buccal epithelium and the initial and 12-mo post-ES blood. The CBL variant was present at reduced VAF in both the ES and 12-mo post-ES peripheral blood samples, at $11 \%$ and $15 \%$, respectively. The CBL was also present in buccal cells at $\sim 2 \%$, a VAF unresolvable by the original initial Sanger analysis.

\section{DISCUSSION}

Trio exome analysis revealed two variants of interest within this proband. The de novo likely pathogenic PPP2R1A variant in the germline was considered clinically significant and felt to account for the proband's clinical features. The PPP2R1A gene encodes the $\alpha$ isoform of the scaffolding subunit of the serine/threonine protein phosphatase type 2A (PP2A) implicated in the negative control of cell growth and division (Janssens and Goris 2001). The subunit consists of 15 tandem repeat loops of HEAT motif (Xu et al. 2006). Alterations in HEAT loops of the scaffolding subunit are associated with $A D$ neurodevelopmental disorder, along with other genes encoding PP2A subunits PPP2R5D, PPP2R5C, PPP2R5B, PPP2CA, and PPP2R2C. PPP2R1A-related disease (OMIM:616362) is characterized by mild to severe intellectual disability, abnormal head size (microcephaly or macrocephaly), hypotonia, severe motor delay, severe speech delay, and brain malformations (corpus callosum hypoplasia, ventriculomegaly, periventricular leukomalacia) (Houge et al. 2015; Lenaerts et al. 2021). Ptosis, hearing loss, ear-shape abnormality, seizures, scoliosis, joint hypermobility, and short stature have also been reported. Previously reported cases with the PPP2R1A variant affecting p.Arg258 showed significant microcephaly, developmental delay, and brain abnormality (hypoplastic corpus callosum and ventriculomegaly), but scoliosis or growth deficiency was not reported (Lenaerts et al. 2021). Craniosynostosis has not been previously reported with PPP2R1A-related disease. Craniosynostosis, progressive scoliosis, and severe growth deficiency seen in our proband may suggest that serine/threonine phosphatase is also involved in osteogenic differentiation (Karkache et al. 2021).

The second variant, a previously described pathogenic somatic variant within the CBL gene, was of uncertain significance in relation to the proband's clinical presentation and of unknown origin. The CBL gene is a proto-oncogene that encodes a RING finger E3 ubiquitin ligase and acts as both a negative regulator of several receptor protein tyrosine kinase signaling pathways by targeting receptors for degradation and as an adaptor protein in tyrosine phosphorylation-dependent signaling (Thien et al. 2001; Fu et al. 2003). The domain structure of the $\mathrm{CBL}$ protein comprises an amino-terminal tyrosine kinase binding (TKB) domain followed by a linker region, RING domain, proline-rich region (PRR), carboxy- 
terminal phosphorylation sites, and ubiquitin association domain (Thien et al. 2001). Pathogenic variation in CBL is described in association with Noonan syndrome-like disorder with or without JMML, characterized by facial dysmorphism, a wide spectrum of cardiac defects, reduced growth, variable cognitive deficits, and musculoskeletal anomalies. Individuals with germline and somatic alterations in the CBL gene have an increased risk of developing JMML (OMIM: 607785), an aggressive myeloproliferative and myelodysplastic neoplasm of early childhood characterized by excessive macrophage/monocyte proliferation (Loh et al. 2009; Niemeyer et al. 2010; Pérez et al. 2010; Pathak et al. 2015).

VAFs $<30 \%$ detected in the setting of germline NGS analysis warrant further evaluation including examination of local sequence context, mapping quality, variant quality, and strand bias, in addition to orthogonal methodologic verification. Variants meeting the aforementioned quality verification may be attributed to varying etiologies including somatic mosaicism, a hematologic disease process, or, commonly, an acquired clonal change in hematopoietic precursor cells (Miller et al. 2020a; Chao et al. 2021). Distinguishing variant etiology is important and has ramifications in relation to diagnosis, surveillance and management, and counseling as related to recurrence risk (such as in the setting of gonadal or gonosomal mosaicism). Clonal hematopoiesis of indeterminate potential (CHIP) is characterized by the detection of acquired alterations in hematopoietic-associated genes in individuals without evidence of hematological malignancy (Heuser et al. 2016; Karner et al. 2019; Steensma and Ebert 2020).

CHIP is associated with an increased risk of development of hematologic neoplasms and generally increases in prevalence with advancing age. The prevalence of CHIP in healthy children is thought to be extremely rare and has not been extensively studied to date in this cohort. CHIP variants have recently been reported to occur at leukemic hotspots in $<1 \%$ of peripheral blood samples from a noncancer pediatric cohort (Feusier et al. 2021). Specific genes have been identified at high risk for acquiring somatic alterations, including TET2, ASXL1, DNMT3A, TP53, SETD2, CHEK2, ATM, NF1, and CBL (Chao et al. 2021).

Of note, the CBL p.Tyr371His variant was not absent from large population databases (gnomAD; Karczewski et al. 2020). Within gnomAD v2.1.1 this variant was present in samples derived from three individuals with ages ranging from 50 to $80 \mathrm{yr}$. It was detected among two exome analyses, and one genome, with retention even among the gnomAD v2.1.1 noncancer data set. Although the genome analysis flagged this variant as failing the random forest filter in gnomAD v2.1.1, gnomAD v3.1.1 displays this as a passing variant. Representative Integrative Genomics Viewer (IGV) images in gnomAD demonstrate that two of the three variants occurred at reduced VAF, thus highlighting the occurrence of somatic variation in population databases.

The importance of CBL to myeloid differentiation and cancer is likely due to its role in numerous signaling pathways and biological functions (Lyle et al. 2019). Heterozygous germline variants in the CBL gene have been detected in unrelated patients with a Noonan syndrome-like disorder with JMML (Loh et al. 2009; Martinelli et al. 2010; Niemeyer et al. 2010; Pérez et al. 2010; Hecht et al. 2020). Within the COSMIC database there are 30 entries for the p.Tyr371His variant (https://cancer.sanger.ac.uk/cosmic; last accessioned October 4, 2021). The histology subtype most frequently reported to harbor the p.Tyr371 His variant is JMML (20), followed by acute myeloid leukemia (3), chronic myelomonocytic leukemia (3), acute leukemia of ambiguous lineage (1), chronic myeloid leukemia (1), myelodysplastic syndrome (1), and an astrocytoma Grade IV (1). The majority of oncogenic alterations in CBL are missense substitutions which inactivate the ubiquitin ligase activity of $\mathrm{CBL}$, while retaining its downstream signaling functions, resulting in oncogenic activation of signaling pathways (Loh et al. 2009; Makishima et al. 2009). Notably, within JMML the p.Tyr371 is a "hotspot" that it is rarely altered in other myeloid malignancies. CBL variants in JMML and myeloid malignancies are associated with somatically acquired isodisomy, resulting in loss of the wild-type allele (Loh et al. 2009). 
The breadth and depth associated with NGS-based ES methodologies enable the potential for detecting somatic variation, and increasingly such variation is recognized in relation to acquired clonal variants from precursor cells (Chao et al. 2021). When a variant is suspected to be somatic in origin, analysis of an additional tissue source is recommended to discern whether the variant is acquired with restriction to a hematopoietic lineage or somatic mosaic in nature. In our study, an additional tissue source was requested (buccal epithelium) to aid in resolving the etiology and clinical significance of the CBL variant. Targeted NGS analysis showed the presence of the CBL variant at $2 \%$ amid the buccal epithelium derived DNA. Although this may represent low-level mosaicism, studies have shown buccal samples contain varying levels of hematopoietic cells, specifically leukocytes (Theda et al. 2018; Chao et al. 2021). We hypothesize that admixed leukocytes amid the collected buccal sample are a likely contributor to the observed VAF, with the most likely explanation that the $C B L$ variant is acquired and restricted to a clonal population of hematopoietic origin. Targeted NGS analysis also showed an increase in the VAF between the blood sample obtained for $E S$ and the sample obtained 12 mo post-ES, at $11 \%$ and $15 \%$, respectively. More data points are needed to discern if this patient exhibits clonal expansion over time.

Our established exome workflow incorporates phenotypic overlap between clinician reported clinical features and disease-association among genes harboring rare variation. Additionally, ClinVar reported pathogenic or likely pathogenic variants are prioritized for review in our pipeline. The CBL variant was present in the proband's exome sequencing results because of phenotypic overlap for global developmental delay and its presence within ClinVar as a pathogenic variant (VCV000013811.5). This variant was further investigated because of reduced VAF. The presence of the de novo likely pathogenic variant in PPP2R1A was thought to account for the proband's clinical presentation as discussed with the clinical geneticist following this patient. Thus, the CBL variant was reported as an incidental finding and targeted sequencing in another tissue source was recommended. Our ES consent includes an option to receive medically actionable variants unrelated to the patient's medical condition, which includes the ACMG 3.0 recommendations (Miller et al. 2021) and any additional genes the laboratory has determined to be likely medically actionable. Although the ACMG 3.0 secondary findings list does not currently include $C B L$, the recent $A C M G$ recommendations surrounding incidental detection of acquired variants does include $C B L$ as one of the genes commonly altered in clonal hematopoiesis (Chao et al. 2021). Notably within our internal ES cohort $(n=780)$ this is the first and only somatic CBL variant to be identified and reported.

Although there are currently no established National Comprehensive Cancer Network (NCCN) screening guidelines surrounding findings within the $C B L$ gene, there are monitoring options for management and surveillance. Regular physical examinations and complete blood counts can be performed in children with CBL syndrome (Villani et al. 2017). Our proband is currently 3 years of age; he exhibits no signs of JMML including hepatosplenomegaly, cytopenias, and absolute monocytosis. At the time of this report, the proband's complete blood count (CBC) remains within normal range, measurement of which was recommended to be obtained every 3 mo per the clinical care team. This finding illustrates that incidental acquired findings may result in additional clinical management and interventions. Our findings highlight the challenges associated with the interpretation of somatic variation and the methodologic and clinical considerations in such instances.

\section{METHODS}

\section{Sequencing, Bioinformatics, and Quality Control}

Proband and parental peripheral blood samples were submitted for clinical ES to The Steve and Cindy Rasmussen Institute for Genomic Medicine at Nationwide Children's Hospital, 
Table 2. Metrics for ES coverage

\begin{tabular}{lcc}
\hline Gene (transcript ID) & Target mean coverage & $\begin{array}{c}\text { Target coverage } 20 \times \text { breadth } \\
( \pm 20 \text { bp padding around } \\
\text { each exonic region) }\end{array}$ \\
\hline PPP2R1A (NM_014225.5) & $189 \times$ & $100 \%$ \\
CBL (NM_005188.3) & $118 \times$ & $100 \%$ \\
\hline
\end{tabular}

Columbus, Ohio. Genomic DNA extraction from peripheral blood and genotyping assay using a custom Agena MassArray panel (Agena) to ensure sample provenance and verify familial relationship was performed as described by Miller et al. (2020a). Libraries were subject to target capture using SureSelect Human All Exon V6 (Agilent) followed by paired-end 151-bp sequencing to $137 \times$ mean depth on a HiSeq 4000 (Illumina), with $96.5 \%$ of targeted bases at $20 \times$ or greater. Metrics for ES coverage specific to PPP2R1A and CBL are shown in Table 2.

Exome sequencing data were demultiplexed using Illumina bcl2fastq Conversion Software. Secondary analysis was performed using Churchill 2.0, which utilizes BWA-MEM to align sequence reads to the reference sequence (GRCh37/hg19 Feb 2009), Picard Tools for deduplication, and GATK Unified Genotyper for single-sample variant calling (Kelly et al. 2015). Mitogen Genetics (Sunquest) was used for annotation and tertiary analysis filtering informed by clinician-provided phenotypes, which were converted into Human Phenotype Ontology (HPO) terms (Köhler et al. 2017).

As standard practice in our exome workflow, BAM files are reviewed for all assessed variants using the IGV (Broad) (Robinson et al. 2011) to manually examine the read count and strandedness, location of the variant within the read, VAF, homology, mapping, and read quality. Genomic regions with known homology as defined by Mandelker et al. (2016) have been incorporated through BED file track into IGV to visually flag the level of homology of the local region. Identity-confirmed parental sequences were also reviewed along with the proband data for side-by-side comparison and the IGV alignment preferences were set to allow for display of coverage allele-fraction threshold at $2 \%$ and retention of soft-clipped reads. Sanger sequencing of de novo and suspected somatic variants was performed on the proband and available parental samples. PCR amplification followed by Sanger sequencing and data analysis was performed as described by Miller et al. (2020a).

The genomic DNA template derived from peripheral blood was added to Platinum SuperFi II PCR Master Mix (Invitrogen) along with CBL primers (forward (5'-GGA AACAAGTCTTCACTTTT-3') and reverse (5'-GCTCAATCTTTACATCCTTATC-3')) and amplified by PCR as previously described (Cottrell et al. 2021). Because of limited residual volume associated with the buccal samples, DNA was incorporated into a $50-\mu \mathrm{L}$ solution of $1 \times$ Platinum SuperFi II PCR Master Mix (Invitrogen) and $20 \mathrm{nM} \mathrm{CBL}$ primers in the sample tube for $10 \mathrm{~min}$ at $37^{\circ} \mathrm{C}$. Following incubation, we performed two $25-\mu \mathrm{L}$ PCR reactions, as outlined above. Finally, products were purified using 1.8 $\times$ SPRIselect and used as DNA template in a second round of PCR. Amplified products were purified using $1.5 \times$ SPRIselect and processed as described (Miller et al. 2020b; Cottrell et al. 2021). Sequencing of the pooled libricons (amplicon libraries; Miller et al. 2020b) occurred on an Illumina iSeq 100. Metrics for targeted NGS coverage specific to CBL are shown in Table 3.

Raw sequencing data were converted to unaligned BAMs by Picard Tools IlluminaBasecallsToSam (v2.19.0) to preserve the unique molecular indentifier (UMI) information. Alignment was performed to the GRCh38 reference using BWA-MEM (v0.7.15) and deduplicated using GATK's MarkDuplicates (v4.1.9), considering both the alignment positions and the UMls to identify duplicates. Base counts of the reads at the variant site were calculated using BAM-read count (v0.8.0) and visually inspected using IGV. 
Competing Interest Statement

The authors have declared no competing interest.

Referees

Tomi Pastinen

Anonymous

Received July 29, 2021; accepted in revised form October 27, 2021.
Table 3. Metrics for targeted next-generation sequencing (NGS)

\begin{tabular}{lccc}
\hline Sample & Total reads & Unique mapping reads & Target mean coverage \\
\hline Blood specimen 1 & $1,818,744$ & 583,440 & $210,143 \times$ \\
Blood specimen 2 & $1,942,102$ & 545,189 & $235,867 \times$ \\
Buccal & $1,589,282$ & 564,599 & $195,879 \times$ \\
\hline
\end{tabular}

\section{Variant Interpretation}

Custom scripting allowed for enrichment of variant attribute data including disease association and phenotype overlap. For each proband, the annotated filtered variant list was evaluated at a consensus case conference attended by clinical laboratory directors, genetic counselors, variant scientists, residents, fellows, and ordering providers when available. Variants that met group consensus were assessed according to the ACMG/AMP guidelines (Richards et al. 2015). Following assessment, variants are reported at the discretion of the clinical laboratory director as either likely causal for proband phenotype or as findings of undetermined clinical relevance to the proband phenotype based upon strength of phenotype overlap with the associated disease.

\section{ADDITIONAL INFORMATION}

\section{Database Deposition and Access}

Variant data have been deposited into the ClinVar database (https://www.ncbi.nlm.nih.gov/ clinvar/) under accession numbers VCV000013811.5 and VCV001738402.1.

\section{Ethics Statement}

Oral consent was obtained from the family for publication. The work presented herein was approved by the institutional review boards (IRBs) designated to oversee and monitor biomedical human subject research at Nationwide Children's Hospital (IRB18-0062).

\section{Acknowledgments}

The authors thank the family described in this manuscript for their support and participation.

\section{Author Contributions}

M.Me., M.T.M., C.E.C., and K.L. conceptualized the study; V.J., M.T.M., C.E.C., and K.L. performed the formal analysis; V.M., C.E.C., and K.L. established the methodology; V.J., S.A.W., and B.J.K. performed the investigation; C.M., M.Mo., and A.E.J.-K. provided resources; M.Me., V.J., and K.L. visualized the project; M.Me., M.T.M., and K.L. wrote the original draft; M.Me., M.T.M., M.Mo., A.E.J.-K., V.M., E.R.M., C.E.C., and K.L. reviewed and edited the manuscript; and M.T.M., C.E.C., and K.L. supervised the project.

\section{REFERENCES}

\footnotetext{
Acuna-Hidalgo R, Bo T, Kwint MP, van de Vorst M, Pinelli M, Veltman JA, Hoischen A, Vissers LELM, Gilissen C. 2015. Post-zygotic point mutations are an underrecognized source of de novo genomic variation. Am J Hum Genet 97: 67-74. doi:10.1016/j.ajhg.2015.05.008

Biesecker LG, Spinner NB. 2013. A genomic view of mosaicism and human disease. Nat Rev Genet 14: 307320. doi:10.1038/nrg3424
} 
Cao Y, Tokita MJ, Chen ES, Ghosh R, Chen T, Feng Y, Gorman E, Gibellini F, Ward PA, Braxton A, et al. 2019. A clinical survey of mosaic single nucleotide variants in disease-causing genes detected by exome sequencing. Genome Med 11: 48. doi:10.1186/s13073-019-0658-2

Chao EC, Astbury C, Deignan JL, Pronold M, Reddi HV, Weitzel JN, ACMG Laboratory Quality Assurance Committee. 2021. Incidental detection of acquired variants in germline genetic and genomic testing: a points to consider statement of the American College of Medical Genetics and Genomics (ACMG). Genet Med 23: 1179-1184. doi:10.1038/s41436-021-01138-5

Clark MM, Stark Z, Farnaes L, Tan TY, White SM, Dimmock D, Kingsmore SF. 2018. Meta-analysis of the diagnostic and clinical utility of genome and exome sequencing and chromosomal microarray in children with suspected genetic diseases. NPJ Genom Med 3: 16. doi:10.1038/s41525-018-0053-8

Cottrell CE, Bender NR, Zimmermann MT, Heusel JW, Corliss M, Evenson MJ, Magrini V, Corsmeier DJ, Avenarius M, Dudley JN, et al. 2021. Somatic PIK3R1 variation as a cause of vascular malformations and overgrowth. Genet Med 23: 1882-1888. doi:10.1038/s41436-021-01211-z

Feusier JE, Arunachalam S, Tashi T, Baker MJ, VanSant-Webb C, Ferdig A, Welm BE, Rodriguez-Flores JL, Ours C, Jorde LB, et al. 2021. Large-scale identification of clonal hematopoiesis and mutations recurrent in blood cancers. Blood Cancer Discov 2: 226-237. doi:10.1158/2643-3230.BCD-20-0094

Fu J-F, Hsu J-J, Tang T-C, Shih L-Y. 2003. Identification of CBL, a proto-oncogene at 11q23.3, as a novel MLL fusion partner in a patient with de novo acute myeloid leukemia. Genes Chromosomes Cancer 37: 214 219. doi:10.1002/gcc.10204

Gajecka M. 2016. Unrevealed mosaicism in the next-generation sequencing era. Mol Genet Genomics 291: 513-530. doi:10.1007/s00438-015-1130-7

Hecht A, Meyer JA, Behnert A, Wong E, Chehab F, Olshen A, Hechmer A, Aftandilian C, Bhat R, Choi SW, et al. 2020. Molecular and phenotypic diversity of CBL-mutated juvenile myelomonocytic leukemia. Haematologica doi:10.3324/haematol.2020.270595

Heuser M, Thol F, Ganser A. 2016. Clonal hematopoiesis of indeterminate potential. Dtsch Arztebl Int 113: 317-322. doi:10.3238/arztebl.2016.0317

Houge G, Haesen D, Vissers LELM, Mehta S, Parker MJ, Wright M, Vogt J, McKee S, Tolmie JL, Cordeiro N, et al. 2015. B56 8 -related protein phosphatase 2A dysfunction identified in patients with intellectual disability. J Clin Invest 125: 3051-3062. doi:10.1172/JCI79860

Janssens V, Goris J. 2001. Protein phosphatase 2A: a highly regulated family of serine/threonine phosphatases implicated in cell growth and signalling. Biochem J 353: 417-439. doi:10.1042/bj3530417

Javadi M, Richmond TD, Huang K, Barber DL. 2013. CBL linker region and RING finger mutations lead to enhanced granulocyte-macrophage colony-stimulating factor (GM-CSF) signaling via elevated levels of JAK2 and LYN. J Biol Chem 288: 19459-19470.

Ji J, Shen L, Bootwalla M, Quindipan C, Tatarinova T, Maglinte DT, Buckley J, Raca G, Saitta SC, Biegel JA, et al. 2019. A semiautomated whole-exome sequencing workflow leads to increased diagnostic yield and identification of novel candidate variants. Cold Spring Harb Mol Case Stud 5: a003756. doi:10 $.1101 /$ mcs.a003756

Kales SC, Ryan PE, Nau MM, Lipkowitz S. 2010. Cbl and human myeloid neoplasms: the Cbl oncogene comes of age. Cancer Res 70: 4789-4794. doi:10.1158/0008-5472.CAN-10-0610

Karczewski KJ, Francioli LC, Tiao G, Cummings BB, MacArthur DG, et al. 2020. The mutational constraint spectrum quantified from variation in 141,456 humans. Nature 581: 434-443. doi:10.1038/s41586-020-2308-7

Karkache IY, Damodaran JR, Molstad DHH, Bradley EW. 2021. Serine/threonine phosphatases in osteoclastogenesis and bone resorption. Gene 771: 145362. doi:10.1016/j.gene.2020.145362

Karner K, George TI, Patel JL. 2019. Current aspects of clonal hematopoiesis: implications for clinical diagnosis. Ann Lab Med 39: 509-514. doi:10.3343/alm.2019.39.6.509

Kelly BJ, Fitch JR, Hu Y, Corsmeier DJ, Zhong H, Wetzel AN, Nordquist RD, Newsom DL, White P. 2015. Churchill: an ultra-fast, deterministic, highly scalable and balanced parallelization strategy for the discovery of human genetic variation in clinical and population-scale genomics. Genome Biol 16: 6. doi:10.1186/s13059-014-0577-x

Köhler S, Vasilevsky NA, Engelstad M, Foster E, Robinson PN, et al. 2017. The human phenotype ontology in 2017. Nucl Acids Res 45: D865-D876. doi:10.1093/nar/gkw1039

Lenaerts L, Reynhout S, Verbinnen I, Laumonnier F, Toutain A, Bonnet-Brillhau F, Hoorne A, Joss S, Chassevant AK, Smith-Hicks C, et al. 2021. The broad phenotypic spectrum of PPP2R1A-related neurodevelopmental disorders correlates with the degree of biochemical dysfunction. Genet Med 23: 352-362. doi:10.1038/ s41436-020-00981-2

Loh ML, Sakai DS, Flotho C, Kang M, Fliegauf M, Archambeault S, Mullighan CG, Chen L, Bergstraesser E, Bueso-Ramos CE, et al. 2009. Mutations in CBL occur frequently in juvenile myelomonocytic leukemia. Blood 114: 1859-1863.

Lupski JR. 2013. Genome mosaicism—one human, multiple genomes. Science 341: 358-359. doi:10.1126/ science. 1239503 
Lyle CL, Belghasem M, Chitalia VC. 2019. c-Cbl: an important regulator and a target in angiogenesis and tumorigenesis. Cells 8: 498. doi:10.3390/cells8050498

Makishima H, Cazzolli H, Szpurka H, Dunbar A, Tiu R, Huh J, Muramatsu H, O’Keefe C, Hsi E, Paquette RL, et al. 2009. Mutations of $\mathrm{E} 3$ ubiquitin ligase $\mathrm{Cb}$ / family members constitute a novel common pathogenic lesion in myeloid malignancies. J Clin Oncol 27: 6109-6116. doi:10.1200/JCO.2009.23.7503

Mandelker D, Schmidt RJ, Ankala A, McDonald Gibson K, Bowser M, Sharma H, Duffy E, Hegde M, Santani A, Lebo M, et al. 2016. Navigating highly homologous genes in a molecular diagnostic setting: a resource for clinical next-generation sequencing. Genet Med 18: 1282-1289. doi:10.1038/gim.2016.58

Martinelli S, De Luca A, Stellacci E, Rossi C, Checquolo S, Lepri F, Caputo V, Silvano M, Buscherini F, Consoli F. et al. 2010. Heterozygous germline mutations in the CBL tumor-suppressor gene cause a Noonan syndrome-like phenotype. Am J Hum Genet 87: 250-257. doi:10.1016/j.ajhg.2010.06.015

Meng L, Pammi M, Saronwala A, Magoulas P, Lalani SR, et al. 2017. Use of exome sequencing for infants in intensive care units: ascertainment of severe single-gene disorders and effect on medical management. JAMA Pediatr 171: e173438. doi:10.1001/jamapediatrics.2017.3438

Miller CR, Lee K, Pfau RB, Reshmi SC, Corsmeier DJ, Hashimoto S, Dave-Wala A, Jayaraman V, Koboldt D, Matthews T, et al. 2020a. Disease-associated mosaic variation in clinical exome sequencing: a two-year pediatric tertiary care experience. Cold Spring Harb Mol Case Stud 6: a005231. doi:10.1101/mcs.a005231

Miller KE, Koboldt DC, Schieffer KM, Bedrosian TA, Crist E, Sheline A, Leraas K, Magrini V, Zhong H, Brennan $P$, et al. 2020b. Somatic SLC35A2 mosaicism correlates with clinical findings in epilepsy brain tissue. Neurol Genet 6: e460. doi:10.1212/NXG.0000000000000460

Miller DT, Lee K, Gordon AS, Amendola LM, Adelman K, Bale SJ, Chung WK, Gollob MH, Harrison SM, Herman GE, et al. 2021. Recommendations for reporting of secondary findings in clinical exome and genome sequencing, 2021 update: a policy statement of the American College of Medical Genetics and Genomics (ACMG). Genet Med 23: 1391-1398. doi:10.1038/s41436-021-01171-4

Niemeyer CM, Kang MW, Shin DH, Furlan I, Erlacher M, Bunin NJ, Bunda S, Finklestein JZ, Gorr TA, Mehta P, et al. 2010. Germline CBL mutations cause developmental abnormalities and predispose to juvenile myelomonocytic leukemia. Nat Genet 42: 794-800. doi:10.1038/ng.641

Pathak A, Pemov A, McMaster ML, Dewan R, Ravichandran S, Pak E, Dutra A, Lee HJ, Vogt A, Zhang X, et al. 2015. Juvenile myelomonocytic leukemia due to a germline CBL Y371C mutation: 35-year follow-up of a large family. Hum Genet 134: 775-787. doi:10.1007/s00439-015-1550-9

Pérez B, Mechinaud F, Galambrun C, Ben Romdhane N, Isidor B, Philip N, Derain-Court J, Cassinat B, Lachenaud J, Kaltenbach S, et al. 2010. Germline mutations of the CBL gene define a new genetic syndrome with predisposition to juvenile myelomonocytic leukaemia. J Med Genet 47: 686-691. doi:10 .1136/jmg.2010.076836

Richards S, Aziz N, Bale S, Bick D, Das S, Gastier-Foster J, Grody WW, Hegde M, Lyon E, Spector E, et al. 2015. Standards and guidelines for the interpretation of sequence variants: a joint consensus recommendation of the American College of Medical Genetics and Genomics and the Association for Molecular Pathology. Genet Med 17: 405-424. doi:10.1038/gim.2015.30

Robinson JT, Thorvaldsdóttir H, Winckler W, Guttman M, Lander ES, Getz G, Mesirov JP. 2011. Integrative Genomics Viewer. Nat Biotechnol 29: 24-26. doi:10.1038/nbt.1754

Steensma DP, Ebert BL. 2020. Clonal hematopoiesis as a model for premalignant changes during aging. Exp Hematol 83: 48-56. doi:10.1016/j.exphem.2019.12.001

Theda C, Hwang SH, Czajko A, Loke YJ, Leong P, Craig JM. 2018. Quantitation of the cellular content of saliva and buccal swab samples. Sci Rep 8: 6944. doi:10.1038/s41598-018-25311-0

Thien CB, Walker F, Langdon WY. 2001. RING finger mutations that abolish c-Cbl-directed polyubiquitination and downregulation of the EGF receptor are insufficient for cell transformation. Mol Cell 7: 355-365. doi:10 .1016/S1097-2765(01)00183-6

Villani A, Greer M-LC, Kalish JM, Nakagawara A, Nathanson KL, Pajtler KW, Pfister SM, Walsh MF, Wasserman JD, Zelley K, et al. 2017. Recommendations for cancer surveillance in individuals with RASopathies and other rare genetic conditions with increased cancer risk. Clin Cancer Res 23: e83-e90. doi:10.1158/1078-0432.CCR-17-0631

Xu Y, Xing Y, Chen Y, Chao Y, Lin Z, Fan E, Yu JW, Strack S, Jeffrey PD, Shi Y. 2006. Structure of the protein phosphatase 2A holoenzyme. Cell 127: 1239-1251. doi:10.1016/j.cell.2006.11.033 


\section{COLD SPRING HARBOR Molecular Case Studies}

\section{Somatic variation as an incidental finding in the pediatric next-generation sequencing era}

Marilena Melas, Mariam T. Mathew, Mari Mori, et al.

Cold Spring Harb Mol Case Stud 2021, 7: a006135 originally published online October 29, 2021 Access the most recent version at doi: $10.1101 /$ mcs.a006135

References This article cites 42 articles, 12 of which can be accessed free at: http://molecularcasestudies.cshlp.org/content/7/6/a006135.full.html\#ref-list-1

License This article is distributed under the terms of the Creative Commons Attribution-NonCommercial License, which permits reuse and redistribution, except for commercial purposes, provided that the original author and source are credited.

Email Alerting Receive free email alerts when new articles cite this article - sign up in the box at the Service top right corner of the article or click here. 